\title{
Locally Optimally-Emitting Clouds and the Variable Broad Emission Line Spectrum of NGC 5548
}

\author{
Kirk T. Korista ${ }^{1,2}$ \\ Department of Physics, Western Michigan University \\ Kalamazoo, MI 49008 \\ korista@wmich.edu \\ and \\ Michael R. Goad ${ }^{3}$ \\ Department of Physics and Astronomy, University of Leicester \\ University Road, Leicester, LE1 7RH, England, UK \\ mrg@star.le.ac.uk
}

\begin{abstract}
In recent work Baldwin et al. proposed that in the geometrically extended broad line regions (BLRs) of quasars and active galactic nuclei (AGN), a range in line-emitting gas properties (e.g., density, column density) might exist at each radius, and showed that under these conditions the broad emission line spectra of these objects may be dominated by selection effects introduced by the atomic physics and general radiative transfer within the large pool of line emitting entities. In this picture, the light we see originates in a vast amalgam of emitters, but is dominated by those emitters best able to reprocess the incident continuum into a particular emission line.

We test this "locally optimally-emitting clouds" (LOC) model against the extensive spectroscopic data base of the Seyfert 1, NGC 5548. The time-averaged, integrated-light UV broad emission line spectrum from the 1993 HST monitoring campaign is reproduced via the optimization of three global geometric parameters: the outer radius, the index controlling the radial cloud covering fraction of the continuum source, and the integrated cloud covering fraction. We make an ad hoc selection from the range of successful models, and for a simple spherical BLR geometry we simulate the emission line
\end{abstract}

\footnotetext{
${ }^{1}$ Department of Physics \& Astronomy, University of Kentucky, Lexington, Ky

${ }^{2}$ Visiting Researcher, School of Physics \& Astronomy, University of St. Andrews, Scotland

${ }^{3}$ School of Physics \& Astronomy, University of St. Andrews, Scotland
} 
light curves for the 1989 IUE and 1993 HST campaigns, using the respective observed UV continuum light curves as drivers. We find good agreement between the predicted and observed light curves and lags - a demonstration of the LOC picture's viability as a means to understanding the BLR environment. Finally, we discuss the next step in developing the LOC picture which involves the marriage of echo-mapping techniques with spectral simulation grids such as those presented here, using the constraints provided by a high quality, temporally well-sampled spectroscopic data set.

Subject headings: galaxies: active - galaxies: individual (NGC 5548) galaxies: nucleigalaxies: Seyfert-line: formation

\section{INTRODUCTION}

An important goal of quasar research is to understand the origin and physics of the gas which reprocesses a substantial fraction of the energy generated by the quasar central engine. Since these broad line-emitting regions (BLRs) cannot (yet) be imaged directly, we must infer their properties from the IR - X-ray spectra. Spectral synthesis codes are one of the necessary tools used in the interpretation of these clues, and sophisticated numerical simulations of the broad emission lines (BELs) of quasars and active galactic nuclei (AGN) sprang into existence some 20 years ago (Davidson 1977; Davidson \& Netzer 1979; Kwan \& Krolik 1981). These were limited to single-slab photoionization calculations, more because of a lack of computer power than because of a lack of observational constraints, though future observations were to better define the breadth of the BLR.

The multi-wavelength monitoring campaigns of the past decade were launched to take advantage of the variable nature of the ionizing continuum and its reverberation signatures in the BELs (Blandford \& McKee 1982). The results of these campaigns demonstrated the existence of compact yet geometrically extended and ionization stratified broad emission line regions whose characteristic sizes scale roughly as $R_{B L R} \approx 0.1 \mathrm{pc}\left(L_{46}\right)^{1 / 2}$ (Peterson 1993; Netzer \& Peterson 1997), where $L_{46}$ is the quasar's mean ionizing luminosity in units of $10^{46} \mathrm{ergs} \mathrm{s}^{-1}$. These and other observations and the increase in computer power spawned a new generation of photoionization models (Rees, Netzer, \& Ferland 1989; Goad, O’Brien, \& Gondhalekar 1993, hereafter GOG93), in which the gas density, column density, and covering fraction were allowed to vary systematically with distance from the continuum source in a geometrically thick BLR. In this scenario each of these parameters are power law functions in radius, meant to mimic a single pressure law governing the conditions of the line emitting gas through the BLR. Most recently Kaspi \& Netzer (1999) applied the pressure law model to their photoionization calculations and took advantage of the constraints provided by the observed integrated flux light curves of five emission lines in the well-studied Seyfert 1, NGC 5548 (Clavel et al. 1991, hereafter C91). Figure 1 shows a plot of its mean UV spectrum from the 1993 HST monitoring campaign. Kaspi \& Netzer concluded that the total hydrogen column densities must be at least $10^{23} \mathrm{~cm}^{-2}$ at a distance of 1 light-day from the continuum source and 
that the hydrogen gas densities here must lie between $10^{11} \lesssim n_{H}\left(\mathrm{~cm}^{-3}\right) \lesssim 10^{12.5}$.

If the properties of the line emitting gas are controlled by a single pressure law, what is it that sets and normalizes it to account for the surprising similarity of quasar/AGN emission line spectra through several orders of magnitude in luminosity? Can more than one "pressure law" exist? Recently, Baldwin et al. (1995) proposed that in a geometrically extended BLR, a range in line-emitting gas properties (e.g., density, column density) might exist at each radius, and showed that under these conditions the BEL spectrum of quasars and AGN may be dominated by selection effects introduced by the atomic physics and general radiative transfer within the large pool of line emitting entities. This model was dubbed "locally optimally-emitting clouds," or LOC. They showed that a typical quasar spectrum results from the summation of this amalgam of clouds, and that ionization stratification and the luminosity-radius relationship that produces the similar spectra are natural outcomes. In the next section we confront the LOC model with the timeaveraged and time-variable spectra of one of the most intensively studied AGN, NGC 5548, to gain further insights into the model's strengths and weaknesses and into the physical characteristics of this object's BLR. In $\S 3$ we discuss the results and mention a new and potentially powerful technique in deriving the physical parameters of the BLR (Horne, Korista, \& Goad 1999) — one that takes the most general approach to the LOC picture. The conclusions are given in $\S 4$.

\section{PUTTING A SIMPLE LOC MODEL TO THE TEST}

Here we will confront the predictions of the LOC model with the observed time-averaged and time-variable BEL spectra of one of the best-studied Seyfert 1 galaxies, NGC 5548 (C91; Korista et al. 1995, hereafter K95; Peterson et al. 1999; references therein). It is not our intention here to derive the line emitting geometry and dynamics of NGC 5548, but rather to test the viability of the LOC model under simple assumptions by comparing the predicted spectrum and emission line light curves with that of a well-studied AGN.

\subsection{Photoionization Grid Computations \& Assumptions}

Using Ferland's spectral synthesis code, Cloudy (v90.04) (Ferland 1997; Ferland et al. 1998) we generated a grid of photoionization models of BEL emitting entities, here assumed to be simple slabs (hereafter "clouds") each of which we assumed has constant gas density and a clear view to the source of ionizing photons. The continuum incident upon the clouds does not include the diffuse emission from other BEL clouds, nor do we consider the effects of cloud-cloud shadowing. The grid dimensions spanned 7 orders of magnitude in total hydrogen gas number density, $7 \leq$ $\log n_{H}\left(\mathrm{~cm}^{-3}\right) \leq 14$, and hydrogen-ionizing photon flux, $17 \leq \log \Phi_{H}\left(\mathrm{~cm}^{-2} \mathrm{~s}^{-1}\right.$ ) $\leq 24$ (see Korista

et al. 1997, hereafter K97), and stepped in 0.125 decade intervals in each dimension (3,249 separate Cloudy models). We will call the plane defined by these two parameters the density - flux plane. For 
the present simulations we assumed all clouds have a single total hydrogen column density, $N_{H}=$ $10^{23} \mathrm{~cm}^{-2}$, though in practice the computations of those clouds with very low ionization parameter, $U_{H} \equiv \Phi_{H} /\left(n_{H} c\right) \lesssim 10^{-5}$, stopped when the electron temperature fell below $4000 \mathrm{~K}$. Below this temperature the gas is mainly neutral and there is very little contribution to the optical/UV emission lines. Each individual spectral simulation was iterated until the hydrogen and helium line optical depths converged to $20 \%$ or better on successive iterations. The emitted spectrum is not all that sensitive to the cloud column density over the range $10^{22} \lesssim N_{H}\left(\mathrm{~cm}^{-2}\right) \lesssim 10^{24}$, since the emitting volumes of most collisional excitation metal lines are fully formed within clouds of column densities $10^{22}-10^{23} \mathrm{~cm}^{-2}$, given a significant range of gas density and ionizing flux (K97; Goad \& Koratkar 1998). However, it should be kept in mind that the variations in an emission line spectrum driven by a variable ionizing continuum can differ significantly for column densities spanning $10^{22}-10^{24} \mathrm{~cm}^{-2}$ (GOG93; Shields, Ferland, \& Peterson 1995; Kaspi \& Netzer 1999). We also assumed that the diffuse emission forms in gas with only thermal motions - this may not be the case and local extra-thermal gas motions could have a significant impact on the diffuse emission spectrum through de-saturation of optically thick lines, altering the radiative transfer, and increasing the contribution of photon pumping to the line emission (Shields, Ferland, \& Peterson 1995). Next, we initially assumed solar gas abundances (Grevesse \& Anders 1989; Grevesse \& Noels 1993); however, for reasons discussed below we altered the gas abundances slightly based upon comparison of the models with the observed time-averaged spectrum of NGC 5548. Finally, we assumed an incident continuum spectral energy distribution (SED) that closely resembles that inferred for NGC 5548 by Walter et al. (1994; model A) using simultaneous IUE and ROSAT/PSPC observations (see also Gondhalekar, Goad, \& O'Brien 1996), and that this continuum is emitted isotropically and does not change shape substantially as the luminosity varies. With an average ionizing photon energy of about $84 \mathrm{eV}$, this continuum is significantly harder than that inferred by Mathews \& Ferland (1987) for typical quasars. This is necessary in order to reproduce the heating per photoionization reflected in the observed mean $\mathrm{C} \mathrm{IV} / \operatorname{Ly} \alpha$ flux ratio $(\sim 1)$, large even for Seyfert 1 spectra.

The equivalent width (EW) contour maps in the density - flux plane for six of the seven UV emission line/line blends considered here are shown in Figure 2a and for Mg II $\lambda 2800$ in Figure 2b. The EW is proportional to the total energy emitted by the line, and so is a measure of the continuum reprocessing efficiency for that emission line. The value of the EW of each point lying in the grid assumes full geometric coverage of the continuum source by that particular cloud. For example, the classical BEL cloud parameters of density and ionizing flux lie at the location of the "star" in the EW contour grids in Figure 2 (e.g., Davidson \& Netzer 1979). The value of the Ly $\alpha$ EW at this location within the density - flux plane is approximately $800 \AA$. It was this predicted EW from a single cloud coupled with the observed EWs of Ly $\alpha$ that led early researchers to deduce the value of the cloud covering fraction in quasars $(\sim 10 \%)$ and Seyfert galaxies $(\sim 20 \%)$. The reader may consult K97 for a brief discussion of the distribution of EW contours for the various emission lines. All EWs in Figure 2 are measured with respect to the incident continuum at $1215 \AA$, and so a ratio of the EW contours of two emission lines yields their flux ratio. Finally, lines of constant $\log U_{H}$ 
run at $45^{\circ}$ angles, from lower left to upper right, in each of density - flux planes in Figure 2.

\subsection{The UV Broad Emission Line Spectrum of NGC 5548}

In this section we derive from the data a time-averaged, rest frame, dereddened, velocityintegrated BEL spectrum of NGC 5548, and then simulate it with a simple LOC model.

\subsubsection{The Time-Averaged Emission Line Spectrum}

Here we establish the velocity-integrated time-averaged ultraviolet BEL spectrum of NGC 5548. Because of its high quality we chose the unweighted mean spectrum from the 1993 HST observing campaign (Figure 1; see also K95). We use an unweighted mean spectrum because we want the average emission line fluxes without regard to differences in $\mathrm{S} / \mathrm{N}$ in the individual spectra. We list the total measured (observed frame, reddened) line fluxes in column 2 of Table 1. Since Mg II $\lambda 2800$ flux was not measured during the 1993 HST campaign, we used a slightly smaller value than its mean flux from the 1989 IUE campaign to reflect the lower mean continuum flux and its expected small response to continuum variations. The measured Mg II flux is also problematic because of its blending with surrounding Fe II emission; see Goad et al. (1999) for a recent deblending analysis for another Seyfert 1. Note that these values are essentially those that appear in column 5 of Table 24 in K95, with the exceptions of C IV and He II + O III]. The direct integrated fluxes of these two sets of emission lines were reported in that table, and those measurements did not include the region of overlap lying between them. Here we use their mean fitted fluxes. Goad \& Koratkar (1998) isolated the UV narrow line spectrum from a single HST spectrum (1992 July; Crenshaw, Boggess, \& Wu 1993) when the continuum and broad emission lines were in a near historic low state (in 1992 April). We list the measured (observed frame, reddened) narrow emission line fluxes in column 3 of Table 1; most are taken from Goad \& Koratkar. The N V value was derived from the analyses in Korista et al. (1995) and that of the Si IV + O IV] blend is our recent estimate. The identification of the narrow line emission contributions of this latter septuplet emission line blend whose individual narrow line widths are expected to be $\approx 5 \AA$ (FWHM) is difficult since their positions in wavelength are spread over $\approx 10 \AA$. Whatever its value, the observations would indicate that the narrow line contribution at $\lambda 1400$ is likely to be small.

Correcting the broad line fluxes for reddening was not straight forward. Galactic H I measurements place $E(B-V)$ near 0.03 (Murphey et al. 1996). However, Kraemer et al. (1998) found an observed narrow emission line ratio of He II $1640 / 4686$ that indicated $E(B-V) \approx 0.07$, placing about $E(B-V) \approx 0.04$ somewhere within NGC 5548. This line ratio is expected to remain near its simple Case B value under most conditions, lying near 7 for conditions in the narrow emission line regions, and thus should be a robust reddening indicator (Seaton 1978; MacAlpine 1981; Ferland et al. 2000). But does this extra reddening lie within the narrow line emitting gas, or in a screen 
covering the narrow and broad emission line regions plus the continuum, or some combination? For conditions present within the broad emission line region, this ratio should lie between 7 and 9 (Ferland et al. 2000), and the observed broad-line ratio might point to the amplitude of the reddening through the sight-line to the broad emission line region. Unfortunately, the isolation of the BEL components of the He II lines is made difficult due to their breadth and blending with other broad lines (Wamsteker et al. 1990). The broad emission line of $\lambda 1640$ is blended with the extreme red wing of C IV and emission from O III] $\lambda \lambda 1661,1666$. An unreported analysis of K95 attempted to isolate the broad He II emission using the rms spectrum as a guide, and found that approximately $2 / 3$ of the broad emission from the He II + O III] blend belonged to He II, though the significance of this finding is difficult to quantify. We have adopted this estimated He II/O III] ratio for the present analysis. On the other hand, we know of no attempt to isolate the BEL component of He II $\lambda 4686$, blended with moderately strong emission from both Fe II and the extreme wing of $\mathrm{H} \beta$. Fortunately, whatever the case may be, the reddening correction is small, and other uncertainties are at least as large. Here we adopt the Galactic reddening value, $E(B-V)=0.03(R(V)=3.1$; extinction curve: Cardelli, Clayton, \& Mathis 1989), to correct the BEL and continuum fluxes, and assume the remaining reddening occurs within the narrow emission line gas of NGC 5548 .

The UV BEL fluxes corrected for narrow line fluxes and Galactic reddening are given in column 4 of Table 1. Finally, column 5 lists the derived time-averaged UV BEL luminosities $\left(H_{\mathrm{o}}=75 \mathrm{~km} \mathrm{~s}^{-1} \mathrm{Mpc}^{-1} ; q_{\mathrm{o}}=0.5 ; z=0.0172\right)$ and their adopted uncertainties (in linear luminosity values, not logarithm values). The latter are rather coarse and meant to merely reflect estimates of the uncertainties associated with the measurements of their narrow and broad line fluxes, but do not reflect the uncertainties associated with the adopted cosmological parameters or reddening/extinction correction.

Finally, for our choice of SED and measured time-averaged value of $\log \lambda L_{\lambda 1350} \approx 43.54$ $\operatorname{ergs~\mathrm {s}^{-1}}$, the hydrogen-ionizing luminosity is $\log L_{i o n} \approx 44.26 \mathrm{ergs} \mathrm{s}^{-1}$. At this luminosity a $\log \Phi_{H}=20 \mathrm{~cm}^{-2} \mathrm{~s}^{-1}$ in Figure 2 corresponds to a distance from the continuum source of $R \approx 12.6\left(75 / H_{\mathrm{o}}\right)$ light-days. It should be kept in mind, however, that because of reverberation effects, the measured energy in the UV continuum is not precisely related to the energy that is measured in the lines, even if the form of the ionizing SED is known. A monitoring campaign should be of sufficient duration such that a given line emitting region has been sampled at least once by the range of Fourier frequency components of the variable incident continuum. Whether such "steady-state" conditions are achievable before non-reverberation (e.g., dynamical) effects alter the line emitting regions is uncertain (see Perry, van Groningen, \& Wanders 1994; Wanders \& Peterson 1996), though the observations do indicate that the reverberation time scales are generally much shorter than the dynamical time scales (Peterson 1993). 


\subsubsection{Simulating the Time-Averaged UV BEL Spectrum}

The first set of assumptions concerning the integration of emission from the clouds in our grid involved the simplification of the geometry: a spherically symmetric distribution of BEL clouds, and we did not consider either the effects of line beaming (Ferland et al. 1992; O'Brien, Goad, \& Gondhalekar 1994) or continuum beaming (Wanders \& Goad 1996).

To derive an integrated emission line spectrum, the spectrum of each cloud lying within the density - flux plane was assigned a weight in two dimensions: gas density and distance from the ionizing source assuming $\Phi_{H} \propto L / R^{2}$. Without specifying the particular shape of the emitting entities, this is equivalent to a two dimensional $\left(n_{H}, R\right)$, spherically symmetric, function in effective "cloud" covering fraction. As in Baldwin et al. (1995) and Ferguson et al. (1997) we made the simplifying assumptions that this function is analytic, separable and a power law in each of the two variables (i.e., $f(R) \propto R^{\Gamma}$ and $g\left(n_{H}\right) \propto n_{H}^{\beta}$; see equations $1 \& 2$ in Ferguson et al.). These assumptions are not central to the LOC model, but were chosen merely for their simplicity given the void of observational constraints. Baldwin (1997) found that composite quasar spectra were best matched if the power law indices for the two weighting functions lay near -1 . This is equivalent to a cloud covering fraction distribution, $C_{f}\left(R, n_{H}\right)$, with equal weighting per decade in the density flux plane. In this case, the emission line EW (i.e., continuum reprocessing efficiency) contours in Figure 2 are also proportional to the emission lines' relative luminosity distributions. Steeper radial and/or flatter gas density cloud distribution functions concentrate the emission at high continuum fluxes and gas densities where the emission is mainly thermalized and inefficient. Flatter radial and/or steeper gas density cloud distributions concentrate the emission at large radii and low gas densities. With minimal line thermalization, the line emission from these latter types of clouds is efficient. However, broad emission line reverberation and line profile studies indicate that significant line luminosity must arise from smaller radii as well (Peterson \& Wandel 1999; Wandel, Peterson, \& Malkan 1999). In this analysis we adopted an index of -1 for the weighting along gas density, but allowed for a range in possible radial covering fraction power law index. The latter was a parameter in the optimization process, explained below.

Next, using the adopted gas density distribution function we summed the emission along the density axis for each radius, producing a radial surface emissivity function for each of the lines and line blends considered (see Figure 3). We considered densities in the range $8 \leq \log n_{H}\left(\mathrm{~cm}^{-3}\right) \leq 12$ at each radius. We did not include in our sum the contributions from transparent clouds with very large $U_{H}$. While low density clouds lying very near to the continuum source may have dimensions that rival their distances from the continuum source, they are also virtually transparent (when Thomson thin) so their existence is irrelevant for the purposes of this study. Clouds with gas densities $n_{H}<10^{8} \mathrm{~cm}^{-3}$ emit unobserved forbidden lines, and our simple constant density, $10^{23} \mathrm{~cm}^{-2}$ column density model clouds become geometrically large relative to the characteristic size of the BLR (C91; Peterson et al. 1991). It is also true that at the distances from the continuum source at which these low density clouds are efficient emitters $\left(\log \Phi_{H} \lesssim 10^{18} \mathrm{~cm}^{-2} \mathrm{~s}^{-1}\right)$, the temperatures of grains, if present, lie below their sublimation points. Netzer \& Laor (1993) suggested grain survival 
as a natural mechanism to cut off the broad emission at large radii; this would serve to demarcate the boundary between the BLR and NLR in AGN. Above gas densities of $10^{12} \mathrm{~cm}^{-3}$, the majority of clouds are mainly continuum emitters, and most of the lines are thermalized, assuming thermal local line widths (Rees, Netzer, \& Ferland 1989; K97). The notable exceptions to this rule are the excited-state recombination lines of $\mathrm{H}$, He I, and He II, which continue to emit efficiently at these high densities (see K97). The radiative transfer of the Balmer lines is probably the least understood and most uncertain of all the prominent AGN emission lines. In addition the general methods employed in codes like Cloudy to determine ionization and thermal equilibria begin breaking down above densities of $10^{12} \mathrm{~cm}^{-3}$. So while gas densities of $\sim 10^{14} \mathrm{~cm}^{-3}$ may be present within the BLR, and may solve the long standing $\mathrm{Ly} \alpha / \mathrm{H} \beta$ problem (recently discussed in Netzer et al. 1995 and Baldwin 1997), we chose not to include this gas in our simulations, nor did we use the Balmer lines to constrain our simulations ${ }^{4}$. The EWs of all emission lines considered here peak at or well below gas densities of $10^{12} \mathrm{~cm}^{-3}$. In summary, the gas density distribution function was fixed and not part of the optimization process.

Finally, we fixed the inner radius of the BLR to 1 light-day. This is not a feature of the LOC picture, and it was done only to accommodate our chosen analytic cloud covering fraction distribution function of physical radii, as appropriate for the luminosity of NGC $5548\left(R \propto\left(L / \Phi_{H}\right)^{1 / 2}\right)$. As long as this choice of inner radius is small its impact on the results is minor, since for the adopted hydrogen-ionizing luminosity the opt-UV emission line gas at smaller radii must be very high density $\left(\gg 10^{12} \mathrm{~cm}^{-3}\right.$ ) and/or very high column density. Otherwise the gas will not emit opt-UV emission lines. In order to account for the response of the emission lines to a variable ionizing continuum, the emission line surface emissivity curves were tabulated down to a radius of about $\frac{1}{3}$ light-day and out to a radius of about $\frac{1}{3}$ pc (Figure 3 ).

To accommodate the fact that several of the measured emission lines are actually blends, we summed the simulated emission from blended species. This obviated the problem of relying heavily upon the results of uncertain deblending analyses. Thus henceforth, Ly $\alpha$ is the sum of $\operatorname{Ly} \alpha \lambda 1216$, He II $\lambda 1216$, and O V] $\lambda 1218$. Si IV is the sum of Si IV $\lambda 1397$, O IV] $\lambda 1402$, and S IV] $\lambda 1405$. He II is the sum of He II $\lambda 1640$, O III] $\lambda 1663$, and $\mathrm{Al}$ II $\lambda 1670$. C III] is a sum of C III] $\lambda 1909$, Si III] $\lambda 1892$, and Al III $\lambda 1860$. N V $\lambda 1240$, C IV $\lambda 1549$, and Mg II $\lambda 2800$ were treated as unblended emission lines. While N V is certainly blended with the red wing of Ly $\alpha$, we assume here that $\mathrm{N} \mathrm{V}$ dominates the measurement.

Using the simulated annealing scheme described in Goad \& Koratkar (1998) to minimize $\chi^{2}$ between the predicted and observed emission line luminosities, we varied the outer radius $\left(R_{\text {out }}\right)$, the

\footnotetext{
${ }^{4}$ With the gas density distribution function and upper limit to the gas density of $10^{12} \mathrm{~cm}^{-3}$ adopted here, the integrated emission from the models presented here find $\mathrm{Ly} \alpha / \mathrm{H} \beta$ ratios of about 25 . This is smaller than the classical high-density Case B ratio of 34 , but still significantly larger than observed, $\sim 11$. Such integrated ratios are attainable with the gas density distribution adopted here and an upper limit to the gas density approaching $10^{14} \mathrm{~cm}^{-3}(\mathrm{Baldwin}$ 1997), but we have excluded the extremely dense gas from consideration here.
} 
power law index on the radial cloud covering fraction ( $\Gamma$; see GOG93), and the normalization to the integrated cloud covering fraction $\left(C_{f}\right)$ to fit the time-averaged BEL spectrum in Table 1 . The first two parameters adjust the relative spectrum and the last normalizes the spectrum to the correct luminosity. For a wide range of combinations of these parameters the emission lines belonging to the $\alpha$-production elements $\mathrm{O}, \mathrm{Si}$, and $\mathrm{Mg}\left(\mathrm{O}\right.$ VI $\lambda 1034^{5}$, O III $\lambda 1663, \mathrm{Si}$ IV + O IV] $\lambda 1400, \mathrm{Si}$ III] $\lambda 1892, \mathrm{Mg}$ II $\lambda 2800)$ were all too strong by factors $1.5-2$ compared to their best estimated observed intensities relative to Ly $\alpha$, He II, and C IV. Note that oxygen and silicon are each represented by a resonance line and a lower ionization intercombination line; each pair of lines together span large regions in the density - flux plane (Figure 2a and K97). As approximate measures of the total heating and photoionization rates, respectively, the intensities of C IV and Ly $\alpha-\mathrm{He}$ II are to first order independent of the gas abundances. For illustrations of various emission line sensitivities to gas abundances in the density - flux plane, see K97 and Korista, Baldwin, \& Ferland (1998). Given the results of this preliminary analyses, we considered the possibility that the gas metal abundances could lie below solar, and we simply scaled the metal/hydrogen abundance ratios to $\frac{1}{2}$ their solar values. However, we left carbon and nitrogen at their solar abundance values, since the few spectral constraints, notably $\mathrm{C} \mathrm{III]} \mathrm{and} \mathrm{N} \mathrm{V,} \mathrm{did} \mathrm{not} \mathrm{indicate} \mathrm{subsolar} \mathrm{abundances} \mathrm{for} \mathrm{these}$ two elements. Carbon, nitrogen, the $\alpha$-elements, and the iron peak elements all have somewhat different stellar population progenitors and need not scale together (e.g., Pagel 1997). The He/H abundance ratio was also left at its solar value. Because the overall metallicity of the simulated gas is slightly sub-solar, the equilibrium electron temperatures within the clouds are slightly elevated over their solar abundance counterparts, and the intensity of the major coolant of the BEL gas, C IV $\lambda 1549$, is enhanced accordingly. This resulted in a generally closer match to the observed Ly $\alpha / C$ IV ratio for our choice of continuum SED. While we ascribe no great significance to these adopted gas abundances, they are less arbitrary than the assumption of solar abundances. A much more complete analysis of parameter space (cloud distribution functions, SEDs) will be required to acquire more accurate measures of the gas abundances.

Figure 4a shows the envelopes in minimum $\chi^{2}$ (solid curves) as functions of each of the three parameters, as determined by the simulated annealing process. The lower dashed lines show the $1 \sigma$ confidence level $\left(\chi^{2}=\chi_{\min }^{2}+1.00=2.01\right)$ for one interesting parameter. The upper dashed lines show the $1 \sigma$ confidence contour $\left(\chi^{2}=\chi_{\min }^{2}+4.72=5.73\right)$ for $N-M=7-3=4$ interesting parameters. We consider all satisfactory models to lie below the upper dashed lines and above the solid curves. Figure $4 \mathrm{~b}$ shows the confidence contours of $\log \chi^{2}$ as a function of $C_{f}$ and $\Gamma$ for fixed values of $\log R_{\text {out }}$ incremented at 0.2 dex. The contours are in steps of 0.25 dex with the outer value equal to 1.50 dex in every case. Satisfactory models lie within the bold dashed contour representing $\chi^{2}=\chi_{\min }^{2}+4.72=5.73$. These appear for outer radii $\log R_{\text {out }} \gtrsim 1.75$. The relative

\footnotetext{
${ }^{5}$ The strength of this line in NGC 5548 has not been reported in the refereed literature. Based upon reports from other Seyfert 1 spectra, we have adopted the ratio O VI/C IV $=0.5$ as an upper limit to its strength. While we did not include this upper limit in the optimization process, we did confirm that this upper limit was realized in all acceptable models for $\Gamma>-1.4$.
} 
emission line spectrum is driven by $\Gamma$ and $R_{\text {out }}$, and it is apparent from the location of the bold contour in Figure 4b that a broad inverse relationship exists between these two parameters. Radial cloud covering fractions which fall off more steeply with radius generally require larger outer radii. This is because some of the emission lines are emissive almost exclusively at larger radii (e.g., C III] and Mg II). Ly $\alpha$ and $\mathrm{C}$ IV are emissive at intermediate and large radii, while N V, He II, and Si IV are also emissive at small radii (see Figures 2 and 3). Since the line emission from clouds at small radii is inefficient for the two strongest and best measured emission lines (Ly $\alpha$ and $\mathrm{C} \mathrm{IV}$ ), larger integrated cloud covering fractions must result from steeper radial covering fraction distributions. The luminosities of $\operatorname{Ly} \alpha$ and C IV and their ratio provide the tightest constraints on the models. Together, Figures $4 \mathrm{a}$ and $4 \mathrm{~b}$ show that satisfactory fits to the time-averaged emission line spectrum are possible for $R_{\text {out }} \gtrsim 60$ light-days, $-1.6 \lesssim \Gamma \lesssim-0.5$, and $0.33 \lesssim C_{f} \lesssim 0.80$, although the condition that $C_{f} \lesssim 50 \%$ (as well as $\mathrm{O}$ VI/C IV $\leq 0.5$ ) constrains $\Gamma \gtrsim-1.4$. These results are not surprising considering the observed intensity of $\mathrm{Mg}$ II and its theoretical EW contours in the density - flux plane, the analysis of Baldwin (1997), and the observed large EW of broad Ly $\alpha$ $(\approx 160 \AA)$, respectively. The models of Ferland et al. (1992), Shields \& Ferland (1993), and Goad \& Koratkar (1998) used a single cloud in their attempts to reproduce the observed properties of Ly $\alpha$ and C IV in NGC 5548, and their required covering fractions exceeded $30 \%-40 \%$. Thus any model that includes additional emission contributions from other clouds for other emission lines must necessarily have a larger covering fraction. Figure 2 also shows that the lowest ionization parameter clouds included in our models $(-5.5 \lesssim \log U(H) \lesssim-4)$ emit little else but Mg II $\lambda 2800$ (plus optical H, He I lines not modeled here; K97).

In choosing one particular fit to the time-averaged spectrum in order to illustrate that model's emission line variability properties, we considered the steepest radial covering fraction index for which an integrated cloud covering fraction $C_{f} \lesssim 50 \%$ resulted: $\Gamma \approx-1.2$. A steep radial cloud covering fraction distribution results in broader distributions in the emission line lags (GOG93), and a broad distribution in lags is observed for NGC 5548. This choice of $\Gamma$ is also in general agreement with that found by Kaspi \& Netzer (1999), -1.33. Figure 4b shows that good solutions with $\Gamma<-1.2$ exist, but at the cost of increasingly larger outer radii and integrated cloud covering fractions. Since we do not know the origin of the emitting gas, the outer radial boundary of the BLR is only loosely constrained from any time-averaged, profile-integrated emission line spectrum. However, a significant contribution of emission from very large radii will dampen the emission line variability amplitudes and in most dynamical models will produce narrow emission lines. Models with integrated cloud covering fractions $C_{f}>50 \%$ are surely affected significantly by cloud-cloud shadowing and diffuse emission from other clouds, and while one could argue that an integrated cloud covering fraction of even $50 \%$ is significant in this sense, we adopted $C_{f} \lesssim 50 \%$ as a reasonable validity limit of these integrated cloud models. These were the only "filters" we applied as we considered our choice of successful model fit to the time-averaged spectrum.

The first three columns of Table 2 lists the emission lines or line blends, their simulated time-averaged luminosities, and their simulated luminosity-weighted radii in light days for the 
model parameters of $R_{\text {out }} \approx 140$ light-days, $\Gamma \approx-1.2$, and an integrated cloud covering fraction $C_{f} \approx 50 \%\left(\chi^{2} \approx 3.33\right)$. If grains were present, their temperatures would lie near or above their sublimation values near this choice of outer radius. All predicted time-averaged line luminosities are within 0.1 dex of their target values (Table 1), based upon the mean spectrum of the 1993 HST campaign. This and similar models predict the following line ratios within the blends: (He II $\lambda 1216+\mathrm{O} \mathrm{V}] \lambda 1218) / \mathrm{Ly} \alpha \lambda 1216 \approx 0.06, \mathrm{O}$ IV $] \lambda 1402 / \mathrm{Si}$ IV $\lambda 1397 \approx 0.5, \mathrm{O}$ III $] \lambda 1663 / \mathrm{He}$ II $\lambda 1640$ $\approx 0.5$, and Si III] $\lambda 1892 / \mathrm{C} \mathrm{III]} \lambda 1909 \approx 0.3$. The emissivity (or luminosity) weighted radius $\left(R_{L}\right)$ is proportional to the emission line response function centroid that in turn is equal to the continuum - emission line cross-correlation function centroid for linear line responses to continuum variations (Koratkar \& Gaskell 1991; GOG93). For this and all satisfactory solutions to the time-averaged spectrum we find the following sequence in increasing $R_{L}: \mathrm{N}$ V, Si IV, He II, C IV, Ly $\alpha, \mathrm{C}$ III], $\mathrm{Mg}$ II. This order very nearly corresponds to the one of increasing emission line lags observed in NGC 5548. Thus a simple LOC model can reproduce the observed spectrum and the general observed trends in ionization stratification within the BLR of this and other objects.

\subsection{Emission Line Light Curves and Lags from an LOC Model}

Using the emission line emissivities, the above adopted model parameters $\left(R_{\text {out }}, \Gamma, C_{f}\right)$, and the simple geometrical assumptions ( $(2.2 .2)$ we computed one dimensional emission line response functions, $\Psi(\tau) \propto \eta(R) R^{2} F(R)$, where $F(R)$ is the emission line surface flux at radius $R, \eta(R)$ is the responsivity of the cloud at radius $R$, and the lag $\tau=\frac{R}{c}(1+\cos \theta)$, with $\theta$ measuring the azimuthal angle. We then convolved these emission line response functions with observed UV $\lambda 1350$ continuum light curves (C91; K95) to generate the emission line light curves and emission line - continuum cross correlation function (CCF) peak and centroid (at 50\% peak) lags (Peterson $\&$ White 1994). We present these light curves and lags here, and discuss their comparisons with the observations in the next section.

Figure 5a shows the comparison between the simulated (emissivity and responsivity-weighted) and observed light curves for Ly $\alpha, \mathrm{N}$ V, Si IV, C IV, He II, C III], and Mg II (recall that several of these lines are blends) from the 1988-1989 AGN Watch IUE campaign for NGC 5548 (C91). Figure 5b shows a similar comparison for the same sets of lines minus Mg II from the 1993 HST campaign (K95). In the latter we also utilized a smoothed version of the measured noisy UV continuum measured by IUE just prior to the HST campaign. The error bars on the observed data points do not reflect the systematic errors present to varying degrees in these data (C91; K95). The model emissivity and responsivity-weighted CCF peak and centroid lags are given in columns 4-7 of Table 2; the measured lags are reported in C91 and K95 and we discuss these further in the next section. The responsivity $\eta(R)$ of an emission line is proportional to the slope $d F_{\text {line }} / d F_{\text {cont }}$ (GOG93), and is a function of radius in our simple spherical BLR. We used the "local" responsivity approximation, in that at every radius each emission line was assigned the local value of the response to a small variation in the continuum flux, given the luminosity/flux normalization for 
the time-averaged spectrum. This is appropriate as long as either the responsivity does not change dramatically with radius or the continuum variations are not too large. While the first assumption does break down at small radii (see Figure 3), these clouds generally do not contribute substantially to the integrated emission line luminosities. Note that we have approximated what should be $\eta\left(R, n_{H}, N_{H}\right)$ as $\eta(R)$. Finally, when generating the simulated emission line light curves, we did not alter the shape of the continuum. The opt-UV continuum in NGC 5548 has been demonstrated to harden with increasing luminosity of the continuum source (e.g., Romano \& Peterson 2000). Marshall et al. (1997) showed that over short time intervals at least the EUV continuum is correlated with but varies with a larger amplitude than does the UV continuum. However, the detailed nature of continuum variability across the energy bands remains a mystery (Nandra et al. 1998). Kaspi \& Netzer tried a variety of different schemes to alter the SED with the UV luminosity in order to produce a better match to the observed light curves. They found that if their adopted SED's EUV break-point energy shifted from 3 Rydbergs to 5 Rydbergs with increasing UV luminosity, their models could better reproduce the He II light curve by increasing this line's variability amplitude. The variability amplitude of Ly $\alpha$ increased as well, which was an improvement, although its mean flux value became too large, and the overall quality of their fits to Ly $\alpha$, C IV, C III, and Mg II diminished.

We emphasize that at no point did we attempt to fit the observed light-curves or lags - we made an ad hoc choice of parameters $\left(R_{\text {out }}, \Gamma, C_{f}\right)$ from a range of solutions which produced a match to the integrated mean emission line spectrum within the uncertainties, and which would result in a reasonable spread in the emission line lags. This was done purposely to test whether or not broad but simple cloud distribution functions which lead to matches to a time-averaged spectrum might also predict the continuum - line reverberation. Additionally, given our present simplistic approach to the LOC picture, we saw no reason to over-fit the data.

\section{DISCUSSION OF RESULTS AND THE FUTURE}

\subsection{Lags}

The emission lines' luminosity-weighted radii in Table 2 can be roughly eye-balled in Figure 2 by mentally centroiding the EW contours (allowing for the integrand limits in gas density and radius), since for $\Gamma=\beta=-1$ the EW contours are directly proportional to those of luminosity. This was pointed out by Baldwin et al. (1995). However, the emission line lags will be biased toward the response of emission line gas from smaller radii which can respond more rapidly and more coherently to the continuum flux variations than gas at larger radii. This explains in part why the predicted emissivity-weighted lags are 3 to 5 times smaller than the corresponding values of $R_{L} / c$ (Pérez, Robinson, \& de la Fuente 1992). The model responsivity-weighted lags will be generally longer than the emissivity-weighted lags because the responsivity $\eta(R)$ is proportional to the slope $d F_{\text {line }} / d F_{\text {cont }}$ which generally flattens at small radii for most lines due to effects of 
ionization and thermalization.

Since the CCF, used to measure the emission line lags, is equivalent to the convolution of the emission line response function with the autocorrelation function of the continuum, the measured lags for an emission line will depend upon the variability nature of the driving continuum, even if the parameters which govern the distribution of emitting gas in phase space are time steady. A continuum variation with a characteristic time scale $\tau_{\text {cont }}$ will most effectively probe line-emitting regions at distances $R \sim c \tau_{\text {cont }} /(1+\cos \theta)$. Differences in the emission line lags are observed between the two campaigns (C91; K95) and predicted in Table 2. These differences may also occur due to a line's luminosity-weighted radius that migrates in and out with the mean ionizing luminosity of the continuum source (O'Brien, Goad, \& Gondhalekar 1995). This is a consequence of non-linearity in the emission line response, and is accommodated to some degree in our locally-linear response approximation. Two other possible reasons for the observed changes in the emission line lags are: (1) the BLR is non-stationary on a time scale of 4 years that separates the campaigns (Wanders \& Peterson 1996), and (2) the finite nature of the monitoring campaigns coupled with the dominance of long time-scale trends in the continuum flux variations (Welsh 1999).

The predicted lags of Ly $\alpha$ and C IV in Table 2 lie fairly near their reported values for the two monitoring campaigns (12 days and $8-16$ days respectively [C91]; $7.5-6.9$ days and $4.6-$ 7.0 days, respectively [K95]). Those of the subordinate lines $\mathrm{N} \mathrm{V}$ and He II are long compared to their observed values ( 4 days and $4-10$ days, respectively [C91]; $1.4-2.4$ days and $1.7-1.8$ days, respectively [K95]), while those of the $\lambda 1900$ blend and $\mathrm{Mg}$ II are too short compared to their observed values $(26-32$ days and $\gtrsim 34$ days, respectively [C91]). The predicted lags of the $\lambda 1400$ blend are too short compared to the measured values from 1989 campaign spectra ( $\gtrsim 12$ days [C91]), and perhaps a bit too long compared to the values measured from the 1993 campaign spectra $(3.5-4.8$ days [K95]). We compare the model and observed light curves in Figures 5a,b, and we will discuss these in more detail in $\S 3.2$. Assuming these differences to be significant, they suggest some clues as to how the actual gas distribution may differ from the one we have derived from the mean spectrum, and we speculate here.

That the observations suggest longer Mg II lags relative to that of Ly $\alpha$ than produced in this model may imply the presence of high density gas at radii larger than our model's outer radius (refer to Figure 2b). Or perhaps the high density gas at larger radii intercepts a larger fraction of the incident continuum than our monotonic radial covering fraction function would predict. This component may be denser than $10^{12} \mathrm{~cm}^{-3}$ and may also be in part the same gas which emits the Balmer emission lines (see K97) not modeled here. We also suggest a possible reason that Kaspi \& Netzer's models generally far underproduced Mg II emission: at the larger radii where this line is emissive, their pressure law resulted in clouds with gas densities that emit Mg II less than optimally. Their imposed outer radius of 100 light-days also did not help in this respect. On the other hand, it is our models' inclusion of this gas that emits Mg II $\lambda 2800$ and little else other than Balmer emission that helps drive the predicted integrated cloud covering fractions to $\gtrsim 40 \%$. It must also be kept in mind that the conditions under which $\mathrm{Mg}$ II $\lambda 2800$ is emitted are affected by 
the radiative transfer of the Balmer lines and continuum, and so is probably the least accurate of the seven line and line blends simulated here.

In most of the models computed here, the lag of the $\lambda 1900$ blend is predicted to be just a bit longer than that of $\operatorname{Ly} \alpha$, whereas the observations show significantly longer lags for the $\lambda 1900$ blend, albeit with considerable uncertainties. A glance at Figure 2a shows that the $\lambda 1900$ blend has a secondary peak in optimal emission near the coordinates $\left(\log n_{H} \approx 9.25, \log \Phi_{H} \approx 19\right)$. This emission is almost entirely that of C III] $\lambda 1909$ (K97) and lies at a far higher ionization parameter than the main diagonal ridge of optimal emission $\left(\log U_{H} \approx-2.5\right)$ for $\mathrm{C}$ III] and the blend. Lying near the $10^{23} \mathrm{~cm}^{-2}$ column density-imposed ionization "cliff," this emission's strength depends much more sensitively to column density — it forms near the back boundary of the cloud for column densities $N_{H} \gtrsim 10^{22} \mathrm{~cm}^{-2}$. Tests show that an integration over a $10^{22} \mathrm{~cm}^{-2}$ column density grid with identical boundary conditions to those here result in a $10 \%$ increase in the luminosityweighted radius of $\lambda 1900$ relative to that of Ly $\alpha$. The presence of a range of column densities, such that the lower gas density clouds (that are emissive in opt-UV lines only at larger radii) have predominantly lower column densities, would further separate the model lags of the $\lambda 1900$ and Ly $\alpha$ lines. All else being equal this would also reduce somewhat the predicted $\lambda 1900$ intensity as well as the $\mathrm{C} \mathrm{III]/Si} \mathrm{III} \mathrm{emission} \mathrm{line} \mathrm{ratio.}$

Finally, it is seen in Figure 2a that the He II blend emission peaks near the $10^{23} \mathrm{~cm}^{-2}$ column density-imposed ionization "cliff" for gas densities $9.5 \lesssim \log n_{H} \lesssim 11.5$. Clouds at smaller radii than this blend's peak in Figure 2a would emit more efficiently in both He II $\lambda 1640$ and O III] $\lambda 1663$ if these clouds had higher column densities. Tests show that an integration over a $10^{24} \mathrm{~cm}^{-2}$ column density grid with identical boundary conditions to those here result in a $15 \%$ decrease in the luminosity-weighted radius of the He II blend relative to that of Ly $\alpha$. The presence of a range of column densities, such that higher column densities were more prevalent for gas densities

$\gtrsim 10^{11} \mathrm{~cm}^{-3}$, would further separate the model lags of the He II blend and Ly $\alpha$. To a smaller extent this would also apply to the N V line as well. These adjustments may not be enough, however, to overcome the significant disparities between the predicted and observed He II blend and $\mathrm{N} \mathrm{V}$ emission line lags with respect to the continuum. The various problems concerning the measured intensities and lags of the He II spectrum in Seyfert 1 galaxies are to be discussed in greater detail in a work in progress (Ferland et al. 2000).

\subsection{Light Curves}

The predicted emission line lags tell only part of the story. As pointed out by Kaspi \& Netzer, the light curves of many emission lines contain far more constraints than either just a mean spectrum or the mean spectrum plus emission line lags. The offsets between the model and observed light curves are for the most part explained by the differences between the model and observed mean broad emission line luminosities (Tables 1 and 2). That the model light curves match as well as they do those from a campaign that occurred 4 years prior to the HST campaign is remarkable 
(Figure 5a). This may mean that the "cloud" parameters are reasonably steady on this time scale (but see Wanders \& Peterson 1996). The responsivity-weighted emission line light curves generally came closer to matching the observed light curves. We invite the reader to compare the results in Figure 5a to those of the more restrictive single pressure law models of Kaspi \& Netzer (1999; Figures 7 and 9) for the five emission lines in common. It should be kept in mind that in the latter work, the uni-dimensional radial power-law cloud parameters were optimized to fit the 5 emission line light curves explicitly.

Disregarding the simple offsets between the model and observed mean luminosities, the greatest mismatches in Figure 5 occur in the variation amplitudes of the two recombination lines, Ly $\alpha$ and He II (blended with O III]), for both campaigns. The model light curves of Kaspi \& Netzer (1999) for these two lines suffered similarly until they allowed for a continuum luminosity dependent $\mathrm{SED}$, as mentioned above. Because of this deficiency the observed inverse correlation between the continuum flux and the $\mathrm{C} \mathrm{IV} / \mathrm{Ly} \alpha$ ratio (Pogge \& Peterson 1992) is not predicted by the model presented here. A variable SED and/or the inclusion of a range of cloud column densities (Shields, Ferland, \& Peterson 1995), as discussed above, may resolve this shortcoming.

\subsection{Future Directions}

The most important feature of the LOC model is its assumption of a large pool of "line emitting entities" from which to draw the emission, and the strong influence of the natural selection effects introduced by the atomic physics and general radiative transfer. While reasonably successful in result, the analysis presented here is actually quite restrictive in its approach to the LOC model. Here and in Baldwin et al. (1995) the cloud parameter distributions were limited to simple power law functions, and the gas density distribution function was fixed over the full breadth of the broad line region. We have also considered only a single column density. Nature need not choose this rigid distribution, and indeed we have discussed how the loosening of some of these assumptions might improve the model's match to the observations. We do not know yet the origin of the "line emitting entities" and the LOC model does not directly address this origin, except that it does not need to be one of finely tuned parameters. The LOC model requires only that there be a wide range of "cloud" properties throughout the BEL geometry. We point out that while the LOC model was developed within the cloud paradigm, other origins for the line emitting matter may fall within its general philosophy. For example, an illuminated wind from an accretion disk (e.g., Murray \& Chiang 1998), while differing in detail, has an illuminating continuum cutting through gradients in gas density and column density within some differential spherical radius. Such gradients will depend upon the angle of the emitting gas above the disk midplane.

The strength in the approach adopted here is in its simplicity in reproducing general emission line properties of both composite quasar spectra (Baldwin et al. 1995) and those of a well observed AGN. However, the derivation of the detailed properties of the broad line emitting regions and thus understanding their true nature will require loosening the simplifying restrictions imposed here. In 
fact one could hope to derive a more general multi-dimensional cloud distribution function whose form need not be analytic, e.g., $f\left(n_{H}, N_{H}, R, \theta, v_{R}\right)$ using time-variable spectra of sufficient quality, where $\theta$ is the azimuthal angle and $v_{R}$ is the radial velocity. Using fake AGN integrated emission line flux and continuum flux light curves and a two-dimensional distribution function $f\left(n_{H}, R\right)$, Horne, Korista, \& Goad (1999) outlined a means to unify the methods of maximum entropy echomapping (Horne, Welsh, \& Peterson 1991; Krolik et al. 1991) with photoionization modeling. In this approach, a general cloud distribution function is constrained mainly by the data and the multidimensional spectral simulation grid, rather than partly by the simplifying analytic assumptions made here. Horne et al. also take into account the effects of anisotropic line emission for simple cloud geometries, and consider a range of symmetric BLR geometries. This new technique combines the direct and indirect methods for solving this complex problem. In later work we will apply this method to the light curves of the 1989 IUE monitoring campaign of NGC 5548, with the hope of learning something concrete about the distribution of the line emitting entities within its broad line region. Eventually we hope to incorporate a more general cloud distribution function, such as $f\left(n_{H}, N_{H}, R, \theta, v_{R}\right)$, in our analysis. However, to take full advantage of this we may need a data set of higher quality (mainly longer duration) than even the 1993 HST campaign. These analyses should impose boundary conditions upon the distribution functions describing the BEL gas, and therefore constrain scenarios for the physical origins and dynamics of this gas.

\section{CONCLUSIONS}

Spanning over 10 billion years of cosmic history and 5 orders of magnitude in energy, the general similarity of quasar/AGN spectra is astounding. Baldwin et al. (1995) suggested the locally optimally-emitting clouds (LOC) picture as a path to advancing our understanding the broad emission lines of AGN: that selection effects (atomic physics and general radiative transfer) operating in a large "pool" of line-emitting environments govern the spectra of quasars. In this picture, the physical characteristics of the line-emitting entities (e.g. gas density, column density) are not unique but are broadly distributed along the radial dimensions of the BLR. The continuum SED and gas chemical abundances are the primary drivers of this natural selection process. In the case of NGC 5548 we find that the ionizing continuum SED must be significantly harder than that of Mathews \& Ferland (1987) to reproduce the observed C IV/Ly $\alpha$ emission line ratio in concurrence with interpolation of contemporaneous multi-wavelength observations, and the gas abundances are roughly solar with some hint that some elements have sub-solar abundances. More accurate derivations of each of these will require analyses beyond the scope of this paper.

Using very simple (power law) "cloud" distribution functions in both radius from the central source and gas density for a fixed cloud column density, we tested the LOC picture against the spectroscopic observations of NGC 5548. For a fixed but broad cloud distribution function in gas density, the outer radius $\left(R_{\text {out }}\right)$, power law index of the radial cloud covering fraction function $(\Gamma)$, and the integrated cloud covering fraction $\left(C_{f}\right)$ were optimized to predict the time-averaged 
UV spectrum from the 1993 HST campaign. Satisfactory fits to the time-averaged emission line spectrum are possible for a broad range of parameters: $R_{\text {out }} \gtrsim 60$ light-days, $-1.6 \lesssim \Gamma \lesssim-0.5$, and $0.33 \lesssim C_{f} \lesssim 0.80$, although the condition that $C_{f} \lesssim 50 \%$ (as well as $\mathrm{O}$ VI/C $\mathrm{IV} \leq 0.5$ ) constrains $\Gamma \gtrsim-1.4$. The maximum outer radius of the BLR is only loosely constrained by a time and velocity-averaged emission line spectrum, but considerations of grain survival at low incident continuum photon fluxes, the breadths of the emission line profiles, and the observed responses of the emission lines probably conspire to limit $R_{\text {out }} \lesssim 200$ light-days. Consideration of the individual emission line light curves would have placed stronger constraints upon $R_{\text {out }}, \Gamma$ and $C_{f}$, had we chosen to do so.

Given that we did not optimize our models to fit directly the light curves of the emission lines, but merely that of the time-averaged spectrum from the 1993 HST campaign, the similarities of the predicted emission line light curves and their lags compared to the observed ones are remarkable. We believe this is a demonstration of the natural predictive power of the LOC picture. The differences between the observed emission line light curves and lags and those predicted by our model suggest to us the following possible general improvements to the LOC model presented here. A range of column densities is present such that higher column density clouds are predominant for higher gas density clouds, while lower column density clouds are predominant for the lower gas density clouds. Because taken together most opt-UV emission lines are visible only for a range in $\bar{U}_{H} \propto \bar{L} /\left(R^{2} n_{H}\right)$ up to some maximum value of $\bar{U}_{H}$, a broadly characteristic radial dependence of column density may be involved. While this is broadly consistent with the findings of Kaspi \& Netzer, there is no reason to believe that such column densities should be uniquely defined at every radius. The gas density distribution function need not remain constant over all radii as assumed here, and in particular there may be a greater concentration of very high density gas at the larger radii than our simple cloud distribution functions would allow.

The next step involves loosening the simplifying constraints imposed here and deriving a general distribution function of cloud properties, e.g., $f\left(n_{H}, N_{H}, R, \theta, v_{R}\right)$, constrained mainly by the observed spectra and the spectral simulations. This can be realized through the marriage of echomapping techniques with spectral simulation grids, using the constraints provided by a high quality temporal spectroscopic data set.

Finally, we wish to respond to a statement that appears in Kaspi \& Netzer (1999). They wrote "Finally, we must comment that present day LOC models are too general and do not contain full treatment of shielding and mixing of the various coexisting components." We are not certain what was meant by "are too general." Being "general" is the main point of the LOC picture. We have shown here and elsewhere the LOC picture has been applied, that there is a fairly wide range in the way that BLR gas can be distributed in $\left(R, n_{H}, N_{H}\right)$ space and still produce spectra that match typical quasar/AGN spectra. Here we fit the mean integrated fluxes of a series of lines in a specific AGN. Many LOC/pressure-law models can do that, but to match the variability requires that the mean formation radius of the lines show a large degree of variation and in such a way that high ionization lines form characteristically closer in to the central ionizing continuum source. As first 
pointed out by Baldwin et al., this appears to be a natural consequence of the LOC picture. By contrast, in the single pressure-law model, a given pressure-law constrains the run of density and column density with radius to vary in a very specific manner, and their starting values must be normalized to a given ionization parameter/incident flux at a pre-defined radius. Amongst other things the LOC picture's generality frees one to investigate some important global parameters over the population of quasars, such as the continuum SED and gas abundances (e.g., Korista, Baldwin, and Ferland 1998). In regards to the "shielding and mixing" comment, the models of broad emission line spectra of AGN presented here, in Baldwin et al. (1995), and elsewhere are and have been internally self-consistent. We discussed a more mature approach to the LOC picture in $\S 3.3$.

We thank an anonymous referee for his/her constructive comments. This work benefited substantially from the support of a PPARC grant of Keith Horne's and we would like to thank Keith and the University of St. Andrews for their hospitality. MRG acknowledges support through a PPARC fellowship during the completion of this work. We are also grateful to Gary Ferland for maintaining his freely distributed code, Cloudy, and to Jack Baldwin for his inspiration. We also thank Jack for his careful reading and suggestions which improved the manuscript. 


\section{REFERENCES}

Baldwin, J.A. 1997, Emission Lines in Active Galaxies: New Methods and Techniques; IAU Colloquium 159., Astronomical Society of the Pacific Conference Series \#113, (San Francisco: PASP), eds. B.M. Peterson, F.-Z. Cheng, and A.S. Wilson.

Baldwin, J.A., Korista, K.T., Ferland, G.J., \& Verner, D.A. 1995, ApJ, 455, L119

Blandford, R.D., \& McKee, C.F. 1982, ApJ, 255, 419

Cardelli, J.A., Clayton, G.C., \& Mathis, J.S. 1989, ApJ, 345, 245

Clavel, J., et al. 1991, ApJ, 366, 64 (C91)

Crenshaw, D.M., Boggess, A., \& Wu, C.C. 1993, ApJ, 416, L67

Davidson, K. 1977, ApJ, 218, 20

Davidson, K., \& Netzer, H. 1979, Rep. Prog. Phys., 51, 715

Ferguson, J.W., Korista, K.T., Baldwin, J.A., \& Ferland, G.J. 1997, ApJ, 487, 122

Ferland, G.J., Peterson, B.M., Horne, K.D., Welsh, W.F., \& Nahar, S.N. 1992, ApJ, 387, 95

Ferland, G.J., 1997, HAZY, A Brief Introduction to Cloudy (Univ. Kentucky Phys. Dept. Int. Rep.)

Ferland, G.J., Korista, K.T., Verner, D.A., Ferguson, J.W., Kingdon, J.B., \& Verner, E.M. 1998, PASP, 110, 761

Ferland, G.J., et al. 2000, in preparation

Goad, M.R., \& Koratkar, A.P. 1998, ApJ, 495, 718

Goad, M.R., Koratkar, A.P., Kim-Quijano, J., Korista, K.T., O’Brien, P.T., \& Axon, D.J. 1999, ApJ, 524, 707

Goad, M.R., O’Brien, P.T., \& Gondhalekar, P.M. 1993, MNRAS, 263, 149 (GOG93)

Gondhalekar, P.M., Goad, M.R., \& O’Brien, P.T. 1996, Ap\&SS, 237, 207

Horne, K.D., Korista, K.T., \& Goad, M.R. 1999, MNRAS, in press

Horne, K., Welsh, W.F., \& Peterson, B.M. 1991, ApJ, L5

Kaspi, S., \& Netzer H. 1999, ApJ, 524, 71

Koratkar, A.P., \& Gaskell, C.M. 1991, ApJ, 375, 85

Korista, K.T., et al. 1995, ApJS, 97, 285 (K95)

Korista, K., Baldwin, J., Ferland, G. 1998, ApJ, 507, 24

Korista, K., Baldwin, J., Ferland, G., Verner, D. 1997, ApJS, 108, 401 (K97)

Kraemer, S.B., Crenshaw, D.M., Filippenko, A.V., \& Peterson, B.M. 1998, ApJ, 499, 719

Krolik, J., Horne, K., Kallman, T.R., Malkan, M.A., Edelson, R.A., \& Kriss, G.A. 1991, ApJ, 371, 541 
Kwan, J., \& Krolik, J. 1981, ApJ, 250, 478

MacAlpine, G.M. 1981, ApJ, 251, 465

Mathews, W.G., \& Ferland, G.J. 1987, ApJ, 323, 456

Murphey, E.M., Lockman, F.J., Laor, A., \& Elvis, M. 1996, ApJS, 105, 369

Murray, N., \& Chiang, J. 1998, ApJ, 494, 125

Nandra, K., Clavel, J., Edelson, R.A., George, I.M., Malkan, M.A., Mushotzky, R.F., Peterson, B.M. Turner, T.J. 1998, ApJ, 505, 594

Netzer, H., \& Laor, A. 1993, ApJ, 404, L51

Netzer, H., Brotherton, M.S., Willis, B.J., Han, M., Wills, D., Baldwin, J.A., Ferland, G.J., \& Brown, I.W.A. 1995, ApJ, 448, 27

Netzer, H., \& Peterson, B.M., 1997, in Astronomical Time Series, eds., D. Maoz, A. Sternberg, \& E. Leibowitz (Dordrecht: Kluwer Academic Publisher), 85

O'Brien, P.T., Goad, M.R., \& Gondhalekar, P.M. 1994, MNRAS, 268, 845

O’Brien, P.T., Goad, M.R., \& Gondhalekar, P.M. 1995, MNRAS, 275, 1125

Pagel, B.E.J., in Nucleosynthesis and Chemical Evolution of Galaxies, (Cambridge University Press: Cambridge, England)

Pérez, E., Robinson, A., \& de la Fuente, L. 1992, MNRAS, 255, 502

Perry, J.J., van Groningen, E., \& Wanders, I. 1994, MNRAS, 271

Peterson, B.M. 1993, PASP, 105, 247

Peterson, B.M., et al. 1991, ApJ, 368, 119

Peterson, B.M., et al. 1999, ApJ, 510, 659

Peterson, B.M., \& Wandel, A. 1999, ApJ, 521, L95

Peterson, B.M., \& White, R.J. 1994, PASP, 106, 879

Pogge, R.W., \& Peterson, B.M. 1992, AJ, 103, 1084

Rees, M.J., Netzer, H., \& Ferland, G.J. 1989, ApJ, 347, 640

Romano, P., \& Peterson, B.M. 2000, ApJ, in press

Shields, J.C., \& Ferland, G.J. 1993, ApJ, 402, 425

Shields, J.C., Ferland, G.J., \& Peterson, B.M. 1995, ApJ, 441, 507

Seaton, M.J. 1978, MNRAS, 185, 5P

Wamsteker, W., et al. 1990, ApJ, 354, 446

Wandel, A., Peterson, B.M., \& Malkan, M.A. 1999, ApJ, 526, 579

Wanders, I., \& Goad, M.R. 1996, ApJ, 469, 113 
Wanders, I., \& Peterson, B.M. 1996, ApJ, 466, 174

Welsh, W.F. 1999, PASP, 111, 1347

This preprint was prepared with the AAS LATEX macros v5.0. 
Fig. 1.- The observed mean UV FOS/HST spectrum of the Seyfert 1 galaxy, NGC 5548. The flux units are $10^{-13} \mathrm{ergs} \mathrm{s}^{-1} \mathrm{~cm}^{-2} \AA^{-1}$. The emission features lying just shortward of the strong broad emission line of Ly $\alpha 1216$ belong to geocoronal Ly $\alpha$.

Fig. 2a.- Contours of $\log W_{\lambda}$ (EW) for six emission lines (or blends), referenced to the incident continuum at $1215 \AA$ for full source coverage, are shown as a function of the hydrogen density and flux of hydrogen-ionizing photons. The total hydrogen column density is $10^{23} \mathrm{~cm}^{-2}$. The EW is in direct proportion to the continuum reprocessing efficiency. The smallest, generally outermost, decade contour corresponds to $1 \AA$, each solid line is 1 decade, and dotted lines represent 0.1 decade steps. The contours generally decrease monotonically from the peak to the $1 \AA$ contour; the solid triangle marks the location of the peak of the dominant line within the blends discussed in the text (Ly $\alpha$, He II, C III], and Si IV). The solid star is a reference point marking the old "standard BLR" parameters.

Fig. 2b.- Same as Figure 2a for the emission line Mg II $\lambda 2800$.

Fig. 3.- The emission line/blend radial surface fluxes from the model clouds for the mean ionizing luminosity, given the adopted weighting function along the gas density axis. The radial distance is measured from the continuum source and the vertical solid line marks 1 light-day.

Fig. 4a.- Envelopes in minimum $\chi^{2}$ (solid curves) as functions of each of the three parameters, as determined by the simulated annealing process. The lower dashed lines show the $1 \sigma$ confidence level $\left(\chi^{2}=\chi_{\text {min }}^{2}+1.00=2.01\right)$ for one interesting parameter. The upper dashed lines show the $1 \sigma$ confidence contour $\left(\chi^{2}=\chi_{\min }^{2}+4.72=5.73\right)$ for $N-M=7-3=4$ interesting parameters.

Fig. 4b. - Confidence contours of $\log \chi^{2}$ as a function of $C_{f}$ and $\Gamma$ for fixed values of $\log R_{\text {out }}$ incremented at 0.2 dex. The contours are in steps of 0.25 dex with the outer value equal to 1.50 dex in every case. Satisfactory models lie within the bold dashed contour representing $\chi^{2}=$ $\chi_{\min }^{2}+4.72=5.73$.

Fig. 5a.- Continuum and broad emission line light curves from the 1989 IUE monitoring campaign of NGC 5548. The solid points with error bars are the observations, the bold curves are the model emissivity weighted predictions and the lighter, generally lower amplitude variation curves are the model responsivity weighted predictions. The vertical axes are in luminosity units: $10^{40}$ $\operatorname{ergs~\mathrm {s}^{-1}} \AA^{-1}$ for the UV continuum and $10^{42} \mathrm{ergs} \mathrm{s}^{-1}$ for the UV emission lines.

Fig. 5b.- Continuum and broad emission line light curves from the 1993 HST monitoring campaign of NGC 5548. The solid points with error bars are the observations, the bold curves are the model emissivity weighted predictions and the lighter, generally lower amplitude variation curves are the model responsivity weighted predictions. The vertical axes are in luminosity units: $10^{40}$ $\operatorname{ergs~\mathrm {s}^{-1}} \AA^{-1}$ for the UV continuum and $10^{42} \mathrm{ergs} \mathrm{s}^{-1}$ for the UV emission lines. 
Table 1. Mean UV Emission Line Strengths in NGC 5548.

\begin{tabular}{lcccc}
\hline \hline \multicolumn{1}{c}{ Emission Line or Blend } & $\begin{array}{c}\text { Observed Total } \\
\text { Flux }\end{array}$ & $\begin{array}{c}\text { Narrow Line } \\
\text { Flux }\end{array}$ & $\begin{array}{c}\text { Corrected BEL } \\
\text { Flux }\end{array}$ & $\log \bar{L}_{B E L}$ \\
\hline Ly $\alpha 1216$ & 694 & 89.5 & 808 & $42.66 \pm 10 \%$ \\
N V $\lambda 1240$ & 79.4 & 6.6 & 96.4 & $41.74 \pm 50 \%$ \\
Si IV + O IV] $\lambda 1400$ & 75.0 & $7.6::$ & 85.6 & $41.69 \pm 30 \%$ \\
C IV $\lambda 1549$ & 676 & 69.7 & 757 & $42.64 \pm 10 \%$ \\
He II $\lambda 1640+$ O III] $\lambda 1663$ & 99.1 & 6.0 & 116 & $41.82 \pm 30 \%$ \\
C III $\lambda 1909+$ Si III] $\lambda 1892$ & 123 & 13.6 & 138 & $41.90 \pm 30 \%$ \\
Mg II $\lambda 2800$ & 129 & $\sim 0$ & 152 & $41.94 \pm 50 \%$ \\
\hline
\end{tabular}

Note. - Only the major components of the line blends are listed. Fluxes are observed frame and given in units of $10^{-14} \mathrm{ergs} \mathrm{s}^{-1} \mathrm{~cm}^{-2}$. Luminosities are given in units of ergs s${ }^{-1}$. All mean values taken from the 1993 HST campaign (K95) with the exception of Mg II. See text for further details. 
Table 2. Model Time-Averaged \& Time-Dependent Properties of the UV Spectrum.

\begin{tabular}{lcccccc}
\hline \hline \multicolumn{1}{c}{ Emission Line or Blend } & \multirow{2}{*}{$\log \bar{L}$} & \multirow{2}{*}{$R_{L} / c$} & \multicolumn{2}{c}{ IUE89 } & \multicolumn{2}{c}{ HST93 } \\
& & & $\tau_{L}$ & $\tau_{\eta}$ & $\tau_{L}$ & $\tau_{\eta}$ \\
\hline Ly $\lambda 1216$ & 42.64 & 41.4 & $10-10.6$ & $10-12.2$ & $7-9.3$ & $8-10.3$ \\
N V $\lambda 1240$ & 41.85 & 12.8 & $3-4.7$ & $5-5.4$ & $3-5.6$ & $4-6.6$ \\
Si IV + O IV] $\lambda 1400$ & 41.70 & 16.0 & $5-5.9$ & $7-8.3$ & $4-6.7$ & $6-8.4$ \\
C IV $\lambda 1549$ & 42.68 & 29.2 & $7-8.8$ & $10-11.5$ & $6-8.3$ & $7-10.2$ \\
He II $\lambda 1640+$ O III] $\lambda 1663$ & 41.74 & 22.1 & $6-6.4$ & $6-7.8$ & $4-7.1$ & $5-8.1$ \\
C III $\lambda 1909+$ Si III] $\lambda 1892$ & 41.82 & 42.3 & $11-13.0$ & $14-16.6$ & $8-10.7$ & $13-12.8$ \\
Mg II $\lambda 2800$ & 41.83 & 67.3 & $15-19.7$ & $21-22.7$ & $15-13.6$ & $17-14.9$ \\
\hline
\end{tabular}

Note. - Only the major components of the line blends are listed. Luminosities are given in units of $\operatorname{ergs~s}^{-1}$. The light travel time to the luminosity-weighted radius, $R_{L} / c$, and the emissivity and responsivity-weighted CCF lags, $\tau_{L}$ and $\tau_{\eta}$, are given in units of days. Each pair of lags in columns $4-7$ is given as "CCF peak - CCF centroid," the latter measured at $50 \%$ of the CCF peak. 


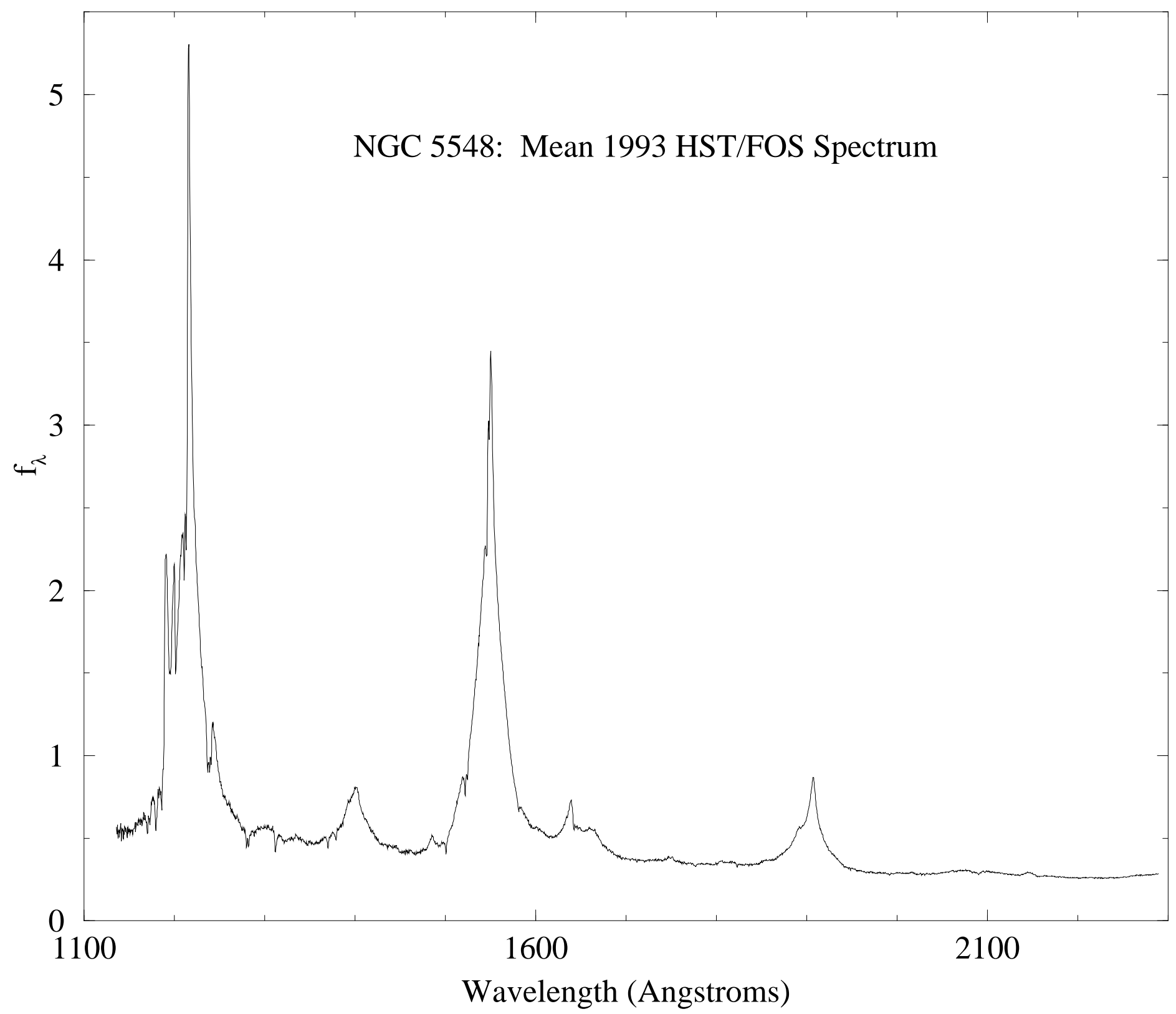




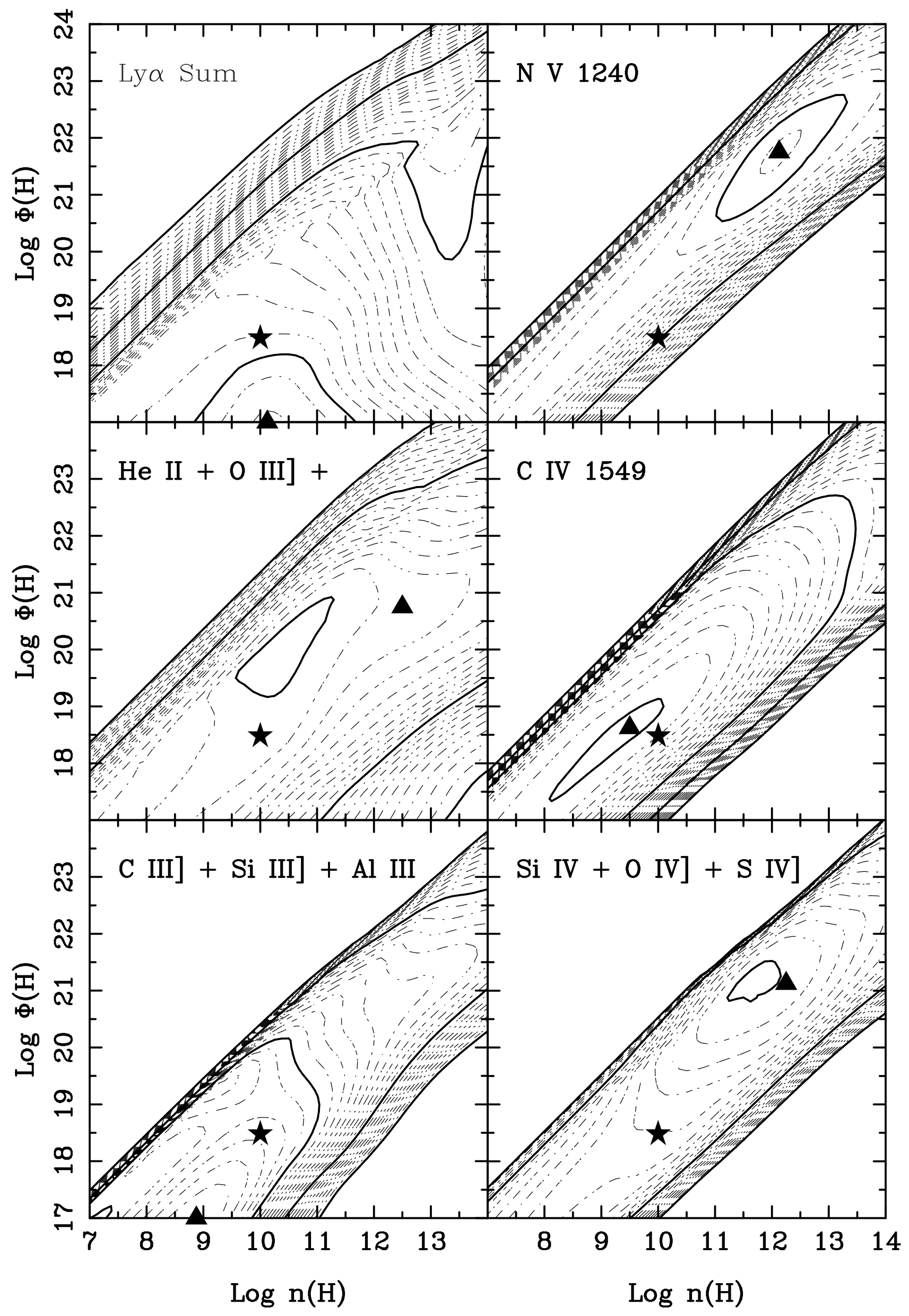




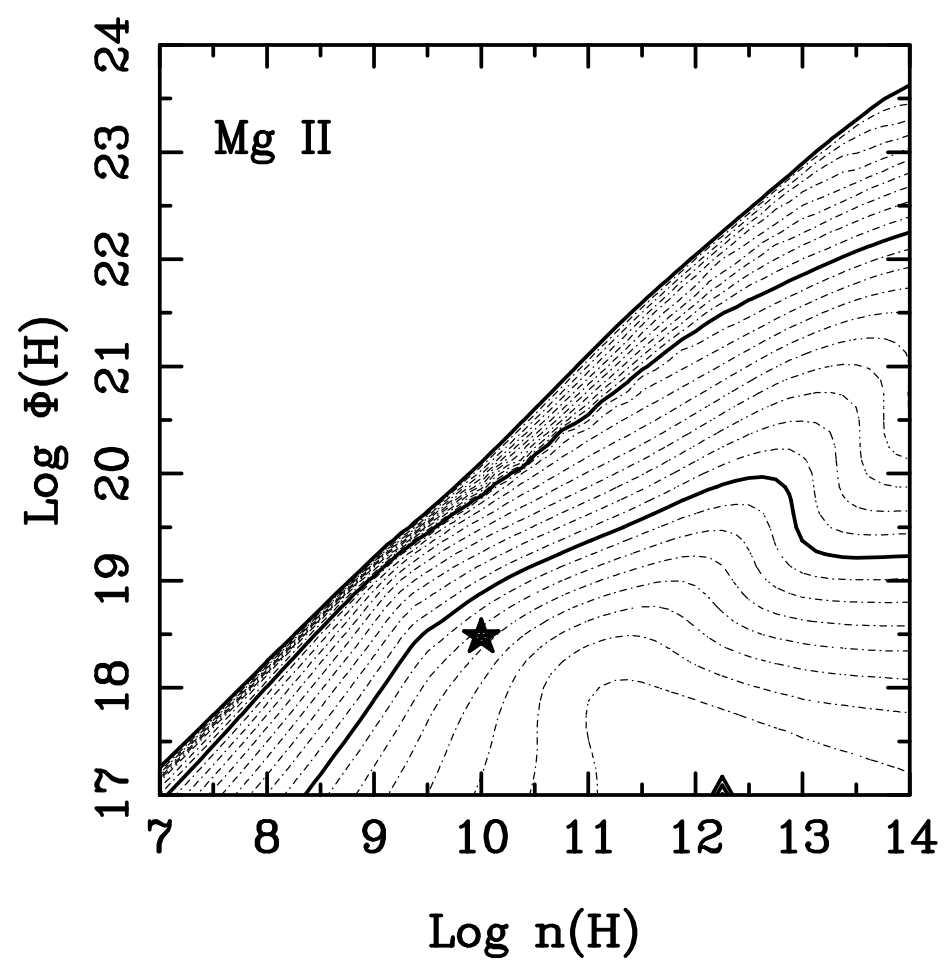




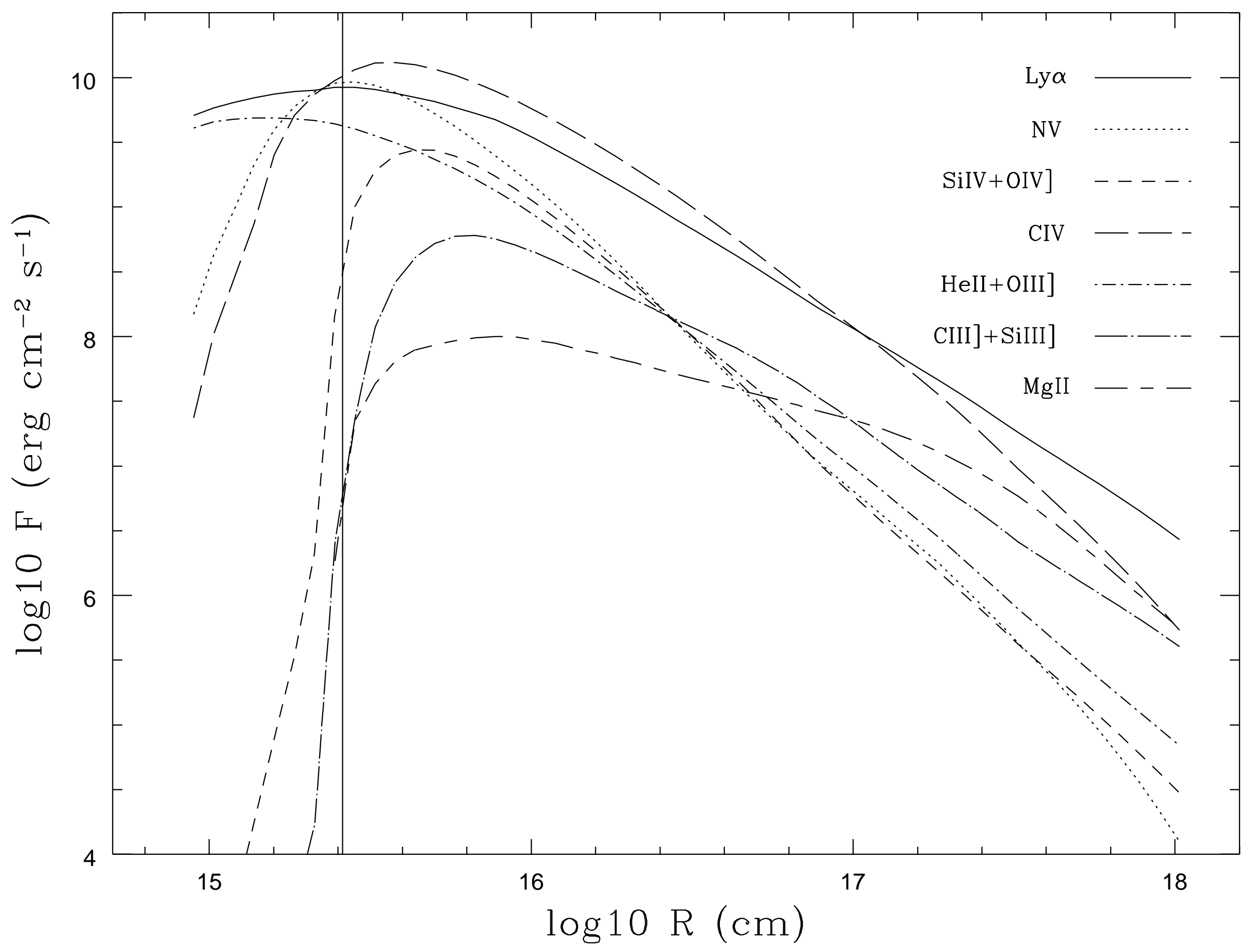



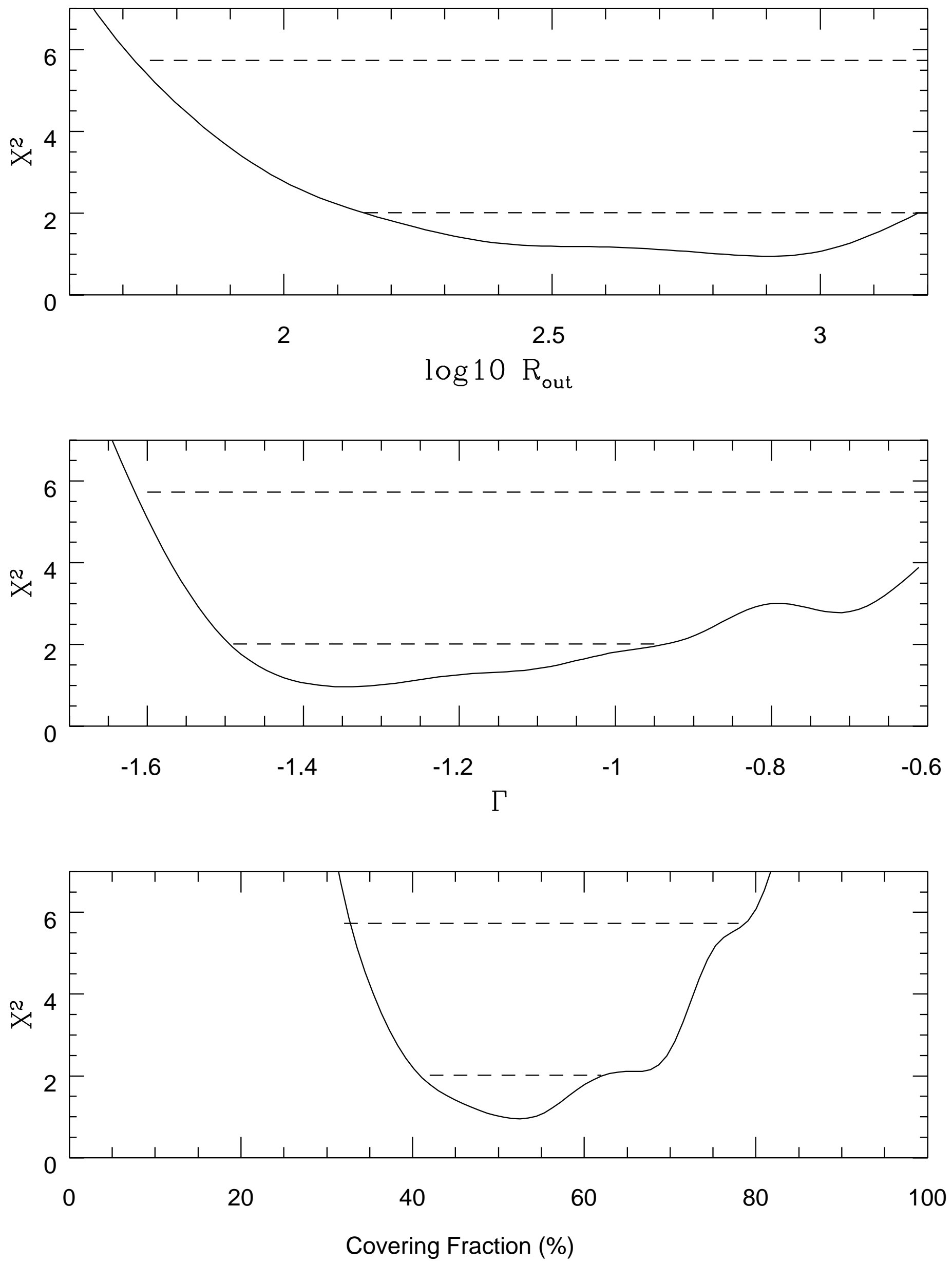


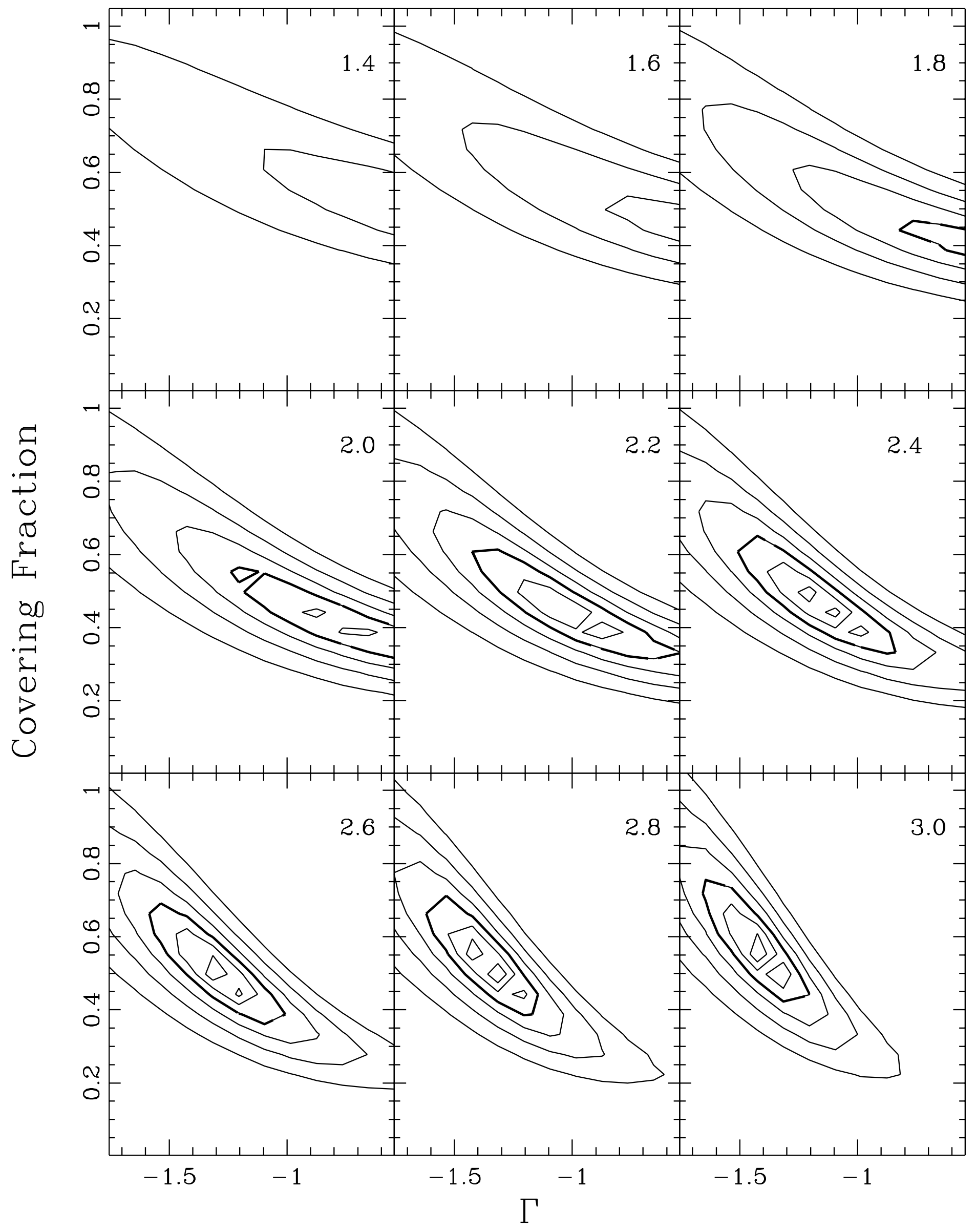



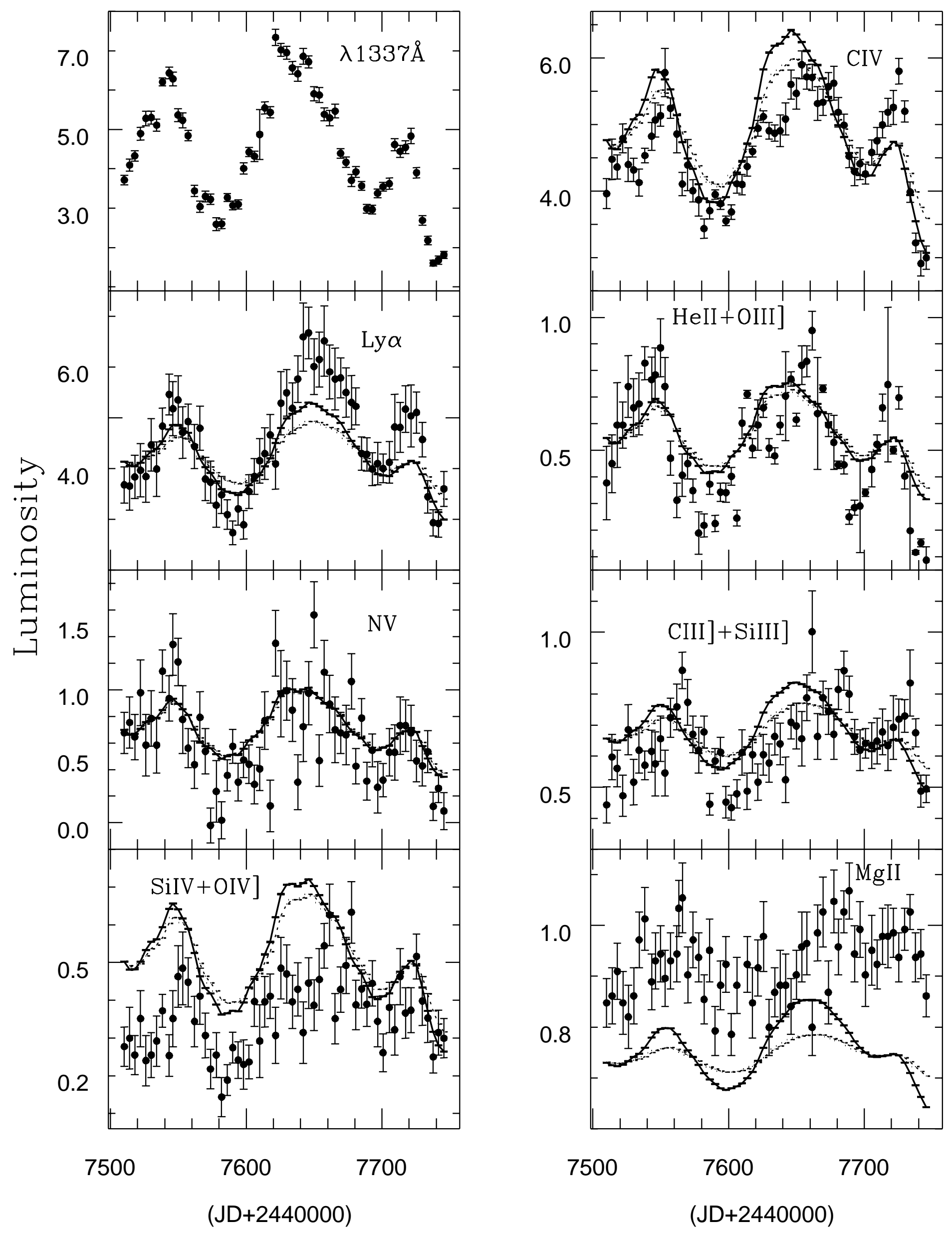


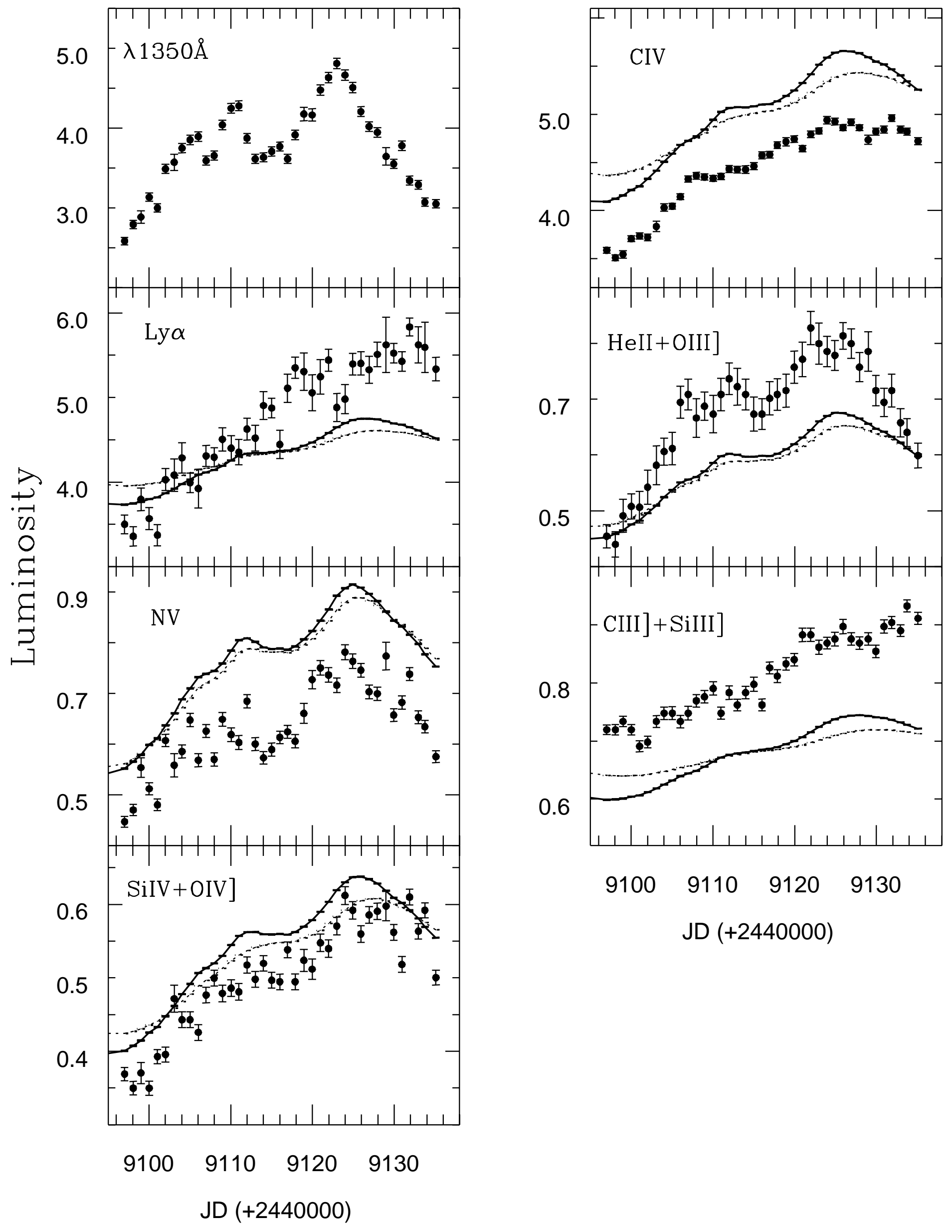

